Bio-grafía. Escritos sobre la Biología y su Enseñanza. ISSN 2027-1034

Número Extraordinario. p.p. $135-144$

Memorias del Primer encuentro ambiental Universidad, ambiente y sustentabilidad: experiencias y prácticas.

\title{
PROPUESTA CURRICULAR PARA LA FORMACIÓN DE COMPETENCIAS PARA EL DESARROLLO SOSTENIBLE EN EL PROGRAMA DE SALUD OCUPACIONAL
}

\section{Luz Alejandra Castillo Rodríguez ${ }^{1}$ \\ Yolanda Ladino Ospina ${ }^{2}$}

\section{Resumen}

La propuesta de la Educación para el Desarrollo Sostenible (EDS) surge principalmente sobre los Objetivos de Desarrollo Sostenible, del Grupo de Trabajo Abierto de la Asamblea General de las Naciones Unidas para la Agenda de Desarrollo post-2015 (2015-30), que da continuidad a los Objetivos de Desarrollo del Milenio (2000-2015); ante esto, el objetivo de este trabajo es la introducción de competencias para el Desarrollo Sostenible en una propuesta curricular de las asignaturas relacionadas con el medio ambiente del programa de salud ocupacional de una Institución de Educación Superior en Bogotá, de acuerdo con lo establecido por la UNESCO, sobre la incorporación de la sostenibilidad en los currículos.

El interés de la inclusión de la educación ambiental al currículo surge de la necesidad de aumentar el campo de acción por parte de las universidades por medio de lo cual se puede garantizar que los profesionales sean formados con una visión global y con los instrumentos y el conocimiento requeridos para encontrar soluciones a los problemas en los entornos en los cuales ellos se desempeñan y realizan su trabajo.

Palabras Clave: Educación para el Desarrollo Sostenibles (EDS), Competencias para el Desarrollo Sostenible en Educación Superior (CDS), Educación Superior, Educación Ambiental (EA), Diseño Curricular.

\footnotetext{
${ }^{1}$ Estudiante de Maestría en Docencia de la Química. Universidad Pedagógica Nacional. Email: alecasro@gmail.com

${ }^{2}$ Profesora Departamento de Química. Universidad Pedagógica Nacional.
} 
Bio-grafía. Escritos sobre la Biología y su Enseñanza. ISSN 2027-1034

Número Extraordinario. p.p. 135-144

Memorias del Primer encuentro ambiental Universidad, ambiente y sustentabilidad: experiencias y prácticas.

\section{Abstract}

The proposal of education for sustainable development arises mainly on the goals of sustainable development, of the Group's work opened in the UN General Assembly of the for the Agenda of development post-2015 (201530 ), which gives continuity to the Millennium Development Goals (20002015); as a result, the objective of this work is the introduction of competencies for sustainable development in a proposed curriculum of subjects related to the environment of the occupational health program of an institution of higher education in Bogotá, in accordance with UNESCO, concerning the incorporation of sustainability into the curriculum.

The interest of the inclusion of it education environmental to the curriculum arises of the need of that increase the field of action from them universities for develop this theme, for ensure them professional are formed with a vision global and with them instruments and the knowledge required for find solutions to them problems in them environments in which they is play and performed his work.

Keywords: Education for Sustainable Development (ESD), Competencies for Sustainable Development (CSD), Higher education, Environmental Education (EE), Curriculum Design.

\section{OBJETIVOS}

\section{General.}

Diseñar una propuesta curricular para la formación de competencias para el desarrollo sostenible en los estudiantes del programa de Tecnología en Salud Ocupacional de una Institución de Educación Superior.

\section{Específicos.}

- Identificar la concepción que tienen de ambiente y su conocimiento previo sobre Educación para la sostenibilidad, por medio de una prueba diagnóstica. 
Bio-grafía. Escritos sobre la Biología y su Enseñanza. ISSN 2027-1034

Número Extraordinario. p.p. 135-144

Memorias del Primer encuentro ambiental Universidad, ambiente y sustentabilidad: experiencias y prácticas.

- Seleccionar los contenidos de una propuesta curricular que involucre situaciones problema de carácter socio- ambiental para fomentar el desarrollo de las competencias para la sostenibilidad.

\section{MARCO TEÓRICO}

La EDS propende por las cuestiones clave de desarrollo sostenible en la enseñanza y el aprendizaje; por ejemplo, el cambio climático, la reducción de desastres, biodiversidad, reducción de la pobreza y el consumo sostenible. También requiere enseñanza participativa y el aprendizaje métodos de motivarán y empoderar a los estudiantes a cambiar su comportamiento y tomar acción para el tema en mención. La EDS, por consiguiente, promueve competencias como pensamiento crítico, imaginando escenarios futuros y tomar decisiones de manera colaborativa (UNESCO, 2014b).

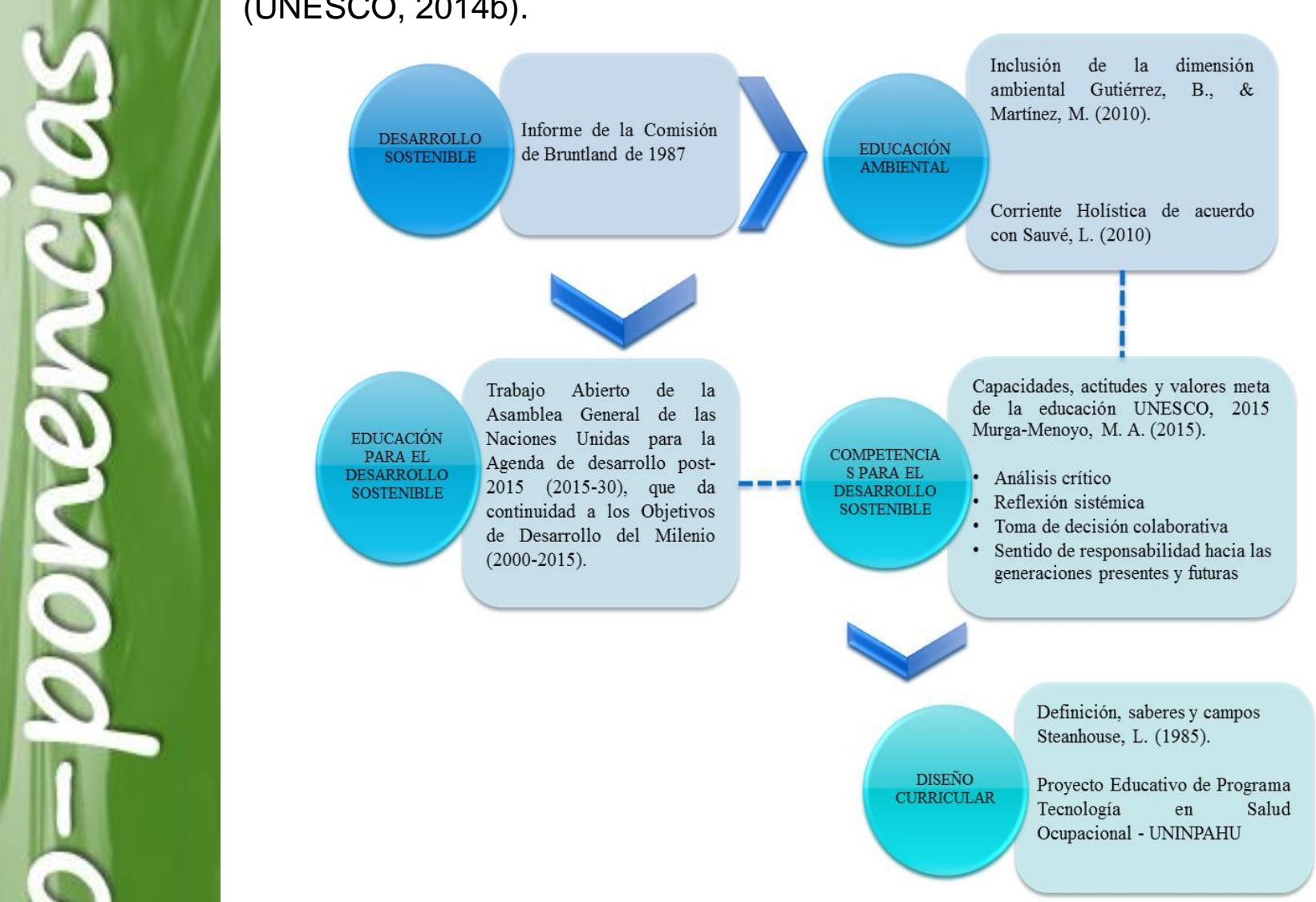

Figura 1. Marco teórico de la investigación 
Bio-grafía. Escritos sobre la Biología y su Enseñanza. ISSN 2027-1034

Número Extraordinario. p.p. $135-144$

Memorias del Primer encuentro ambiental Universidad, ambiente y sustentabilidad: experiencias y prácticas.

\section{METODOLOGÍA}

La perspectiva de esta investigación es cualitativa con un enfoque cuasi experimental sin grupo control, esto debido a que no hay un control de las variables de selección y el estudio surge como una de las posibles alternativas. Para el desarrollo de ésta se toma como población general el espacio académico de salud ambiental de un programa de tecnología en salud ocupacional perteneciente a la decanatura de la facultad de ciencias económicas y administrativas de una Institución de Educación Superior, la muestra pertenece a los estudiantes de dos grupos y los docentes del programa.

Para el desarrollo de los objetivos de la investigación se proponen las siguientes fases:

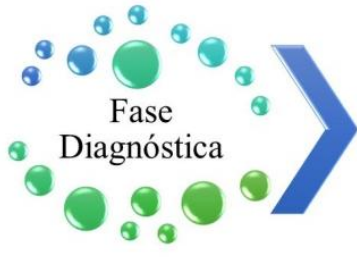

Caracterización de la población para conocer las concepciones que tienen los estudiantes y profesores sobre la Educación Ambiental.

\section{Fase de identificación de Concepciones de Educación Ambiental}

Aproximación a una concepción de educación ambiental, aquella que se ajusta el perfil profesional

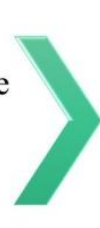

Fase de desarrollo de la propuesta curricular

\section{Propuesta} curricular,

inclusión de problemas socio ecológicos de acuerdo con las competencias

$$
\text { para }
$$

desarrollo sostenible

el

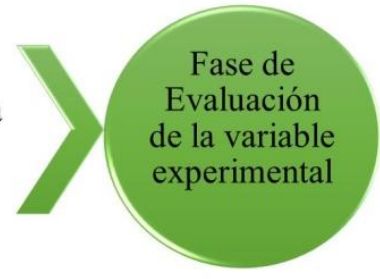

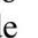

Figura 2. Fases de la Investigación. Fuente: Autor

\section{RESULTADOS}

El análisis crítico y la reflexión sistémica se han seleccionado en consonancia con los temas y de acuerdo a los perfiles profesionales de una institución y se ha establecido una matriz de competencias que serán alcanzadas por medio de la propuesta curricular. 
Bio-grafía. Escritos sobre la Biología y su Enseñanza. ISSN 2027-1034

Número Extraordinario. p.p. 135-144

Memorias del Primer encuentro ambiental Universidad, ambiente y sustentabilidad: experiencias y prácticas.

Para la formación en estas CDS se ha requerido hacer un análisis de la relación directa entre los temas y los perfiles profesionales. Para esto se identificaron las concepciones que se tienen de la educación ambiental para luego poder hacer una aproximación a una concepción/corriente a la que se ajusta el perfil profesional de la población objeto de estudio de acuerdo a Sauvé (2010).

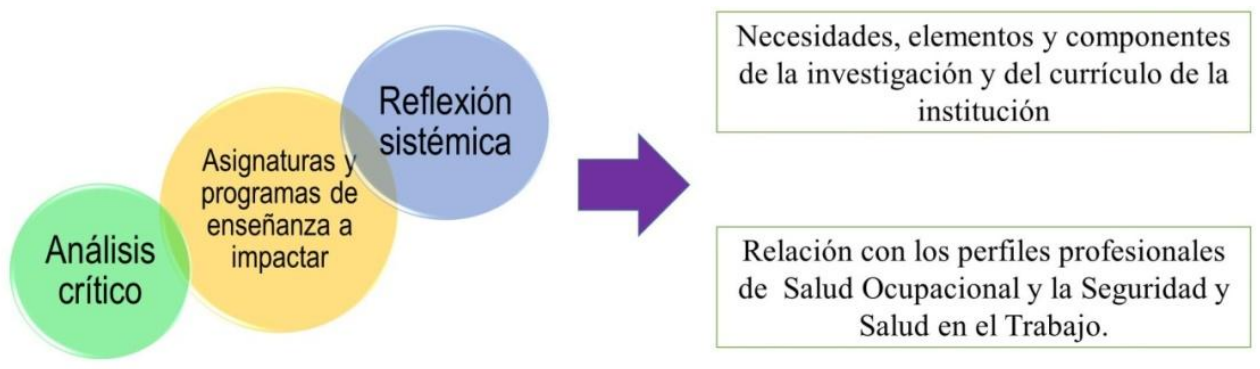

Las competencias para la sostenibilidad seleccionadas de acuerdo a los perfiles profesionales se han elaborado teniendo como base lo establecido por (Murga-Menoyo, 2015), de acuerdo a la matriz básica de competencias para la sostenibilidad:

\section{Análisis Crítico - (Pensamiento crítico y compromiso ético e intelectual)}

- Comprender que el conocimiento es incompleto y está teñido de subjetividad

- Comprender que todo sistema (conceptual, socioeconómico, etc.) presenta disfunciones que pueden ser identificadas y corregidas

- Reconocer las disfunciones sociales y económicas que se oponen al desarrollo sostenible

- Proponer alternativas de mejora

II. Reflexión sistémica - (Pensamiento relacional y holístico)

- Comprender la realidad, física y social, como un sistema dinámico de factores interrelacionados, a nivel global y local

- Comprender las interrelaciones entre valores, actitudes, usos y costumbres sociales, estilos de vida

- Profundizar en las causas de los fenómenos, hechos y problemas 
Bio-grafía. Escritos sobre la Biología y su Enseñanza. ISSN 2027-1034

Número Extraordinario. p.p. $135-144$

Memorias del Primer encuentro ambiental Universidad, ambiente y sustentabilidad: experiencias y prácticas.

- Comprender al ser humano como un ser ecodependiente

Teniendo en cuenta lo anterior es necesario hacer una identificación de los elementos que constituyen el currículo de acuerdo a los temas y perfiles profesionales, teniendo como punto de referencia dos dimensiones: conocer y comprender; y saber actuar para cada una de las CDS, tal y como se explica en la Tabla 1.

Tabla 1. Matriz de Competencias para el Desarrollo Sostenible en la IES

Dimensiones

\begin{tabular}{|c|c|c|c|c|}
\hline \multirow[b]{2}{*}{$\begin{array}{c}\text { Competen } \\
\text { cia }\end{array}$} & \multirow[b]{2}{*}{$\begin{array}{c}\text { Compon } \\
\text { entes }\end{array}$} & \multirow[b]{2}{*}{ Capacidad para... } & \multirow{2}{*}{\multicolumn{2}{|c|}{$\begin{array}{ll}\begin{array}{l}\text { Conocer y } \\
\text { comprender }\end{array} & \text { Saber actuar } \\
\text { (Conocimiento } & \text { (Aplicación práctica } \\
\text { teórico de un campo y operativa } \\
\text { académico, la } & \text { del conocimiento y } \\
\text { capacidad de } & \text { los valores a } \\
\text { conocer } & \text { ciertas situaciones) } \\
\text { y comprender) } & \end{array}$}} \\
\hline & & & & \\
\hline $\begin{array}{l}\text { Reflexión } \\
\text { Sistémica }\end{array}$ & 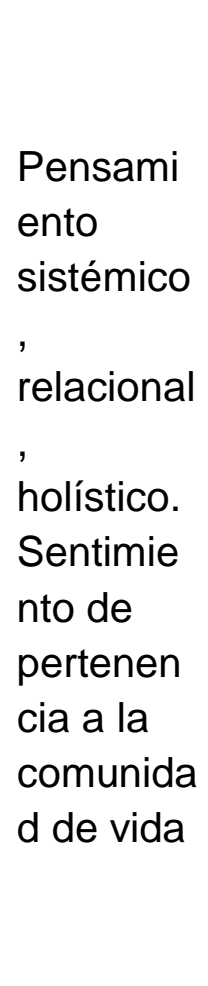 & $\begin{array}{l}\text { - Comprender la } \\
\text { realidad, física y } \\
\text { social, como un } \\
\text { sistema dinámico, a } \\
\text { nivel global (macro) } \\
\text { y local (micro). } \\
\text {-Comprender las } \\
\text { interrelaciones entre } \\
\text { valores, actitudes, } \\
\text { usos y costumbres } \\
\text { sociales, estilos de } \\
\text { vida; y de todos } \\
\text { ellos con las } \\
\text { problemáticas } \\
\text { socioambientales }\end{array}$ & $\begin{array}{l}\text { Crea las } \\
\text { condiciones para el } \\
\text { pensamiento crítico } \\
\text { en el entorno } \\
\text { escolar para } \\
\text { cuestionarse } \\
\text { asunciones y } \\
\text { reconocer y } \\
\text { respetar diferentes } \\
\text { tendencias en } \\
\text { diferentes } \\
\text { situaciones. } \\
\text { De acuerdo con } \\
\text { esto es necesario } \\
\text { considerar las } \\
\text { concepciones que } \\
\text { se tienen acerca de } \\
\text { la educación }\end{array}$ & $\begin{array}{l}\text { Desarrollo de } \\
\text { competencias que } \\
\text { les permitan } \\
\text { entender y conocer } \\
\text { cuáles son los } \\
\text { efectos positivos y } \\
\text { negativos, internos } \\
\text { y externos del } \\
\text { ambiente en la } \\
\text { situación de } \\
\text { trabajo, la } \\
\text { organización del } \\
\text { trabajo y la salud } \\
\text { del trabajador, así } \\
\text { como su impacto } \\
\text { en el entorno y la } \\
\text { comunidad. } \\
\text { Enumera las }\end{array}$ \\
\hline
\end{tabular}


Bio-grafía. Escritos sobre la Biología y su Enseñanza. ISSN 2027-1034

Número Extraordinario. p.p. 135-144

Memorias del Primer encuentro ambiental Universidad, ambiente y sustentabilidad: experiencias y prácticas.

\begin{tabular}{|lll|}
\hline ambiental, & interrelaciones \\
ambiente, gestión & entre valores, \\
ambiental, & actitudes, usos y \\
normatividad & costumbres \\
ambiental, factores & sociales, estilos de \\
de riesgo & vida de acuerdo a \\
ambientales. & la salud \\
& ocupacional y la \\
Analiza & seguridad \\
características de & industrial, \\
los procesos & encaminado al \\
productivos y & desarrollo de \\
riesgos generados & análisis de \\
desde la & accidentes de \\
concepción de & trabajo y planes de \\
ambiente & emergencia \\
& acordes a la \\
& comunidad \\
\hline
\end{tabular}


Bio-grafía. Escritos sobre la Biología y su Enseñanza. ISSN 2027-1034

Número Extraordinario. p.p. $135-144$

Memorias del Primer encuentro ambiental Universidad, ambiente y sustentabilidad: experiencias y prácticas.

\begin{tabular}{|c|c|c|c|c|}
\hline $\begin{array}{c}\text { Análisis } \\
\text { Crítico }\end{array}$ & $\begin{array}{l}\text { Pensami } \\
\text { ento } \\
\text { crítico, } \\
\text { compromi } \\
\text { so ético, } \\
\text { compromi } \\
\text { so } \\
\text { intelectua } \\
\text { I }\end{array}$ & $\begin{array}{l}\text { Comprender que } \\
\text { todo paradigma, } \\
\text { enfoque o punto de } \\
\text { vista está sujeto a } \\
\text { coordenadas } \\
\text { temporales, } \\
\text { culturales, } \\
\text { económicas, } \\
\text { emocionales, etc. } \\
\text { Comprender que } \\
\text { todo sistema } \\
\text { (conceptual, } \\
\text { socioeconómico, } \\
\text { etc.) presenta } \\
\text { disfunciones que } \\
\text { pueden ser } \\
\text { identificadas y } \\
\text { corregidas }\end{array}$ & $\begin{array}{l}\text { Propone medidas } \\
\text { preventivas para } \\
\text { controlar factores } \\
\text { de riesgo dirigido a } \\
\text { disminuir daños al } \\
\text { trabajador, } \\
\text { comunidad y } \\
\text { ambiente } \\
\text { Propone medidas } \\
\text { de control para } \\
\text { factores de riesgo } \\
\text { generados desde y } \\
\text { para el ambiente }\end{array}$ & $\begin{array}{l}\text { Gestiona procesos } \\
\text { de Promoción y } \\
\text { Prevención } \\
\text { manteniendo el } \\
\text { equilibrio de los } \\
\text { ecosistemas } \\
\text { haciendo uso de } \\
\text { las herramientas } \\
\text { propias de la } \\
\text { gestión ambiental. }\end{array}$ \\
\hline
\end{tabular}

Fuente: Autor

\section{CONCLUSIONES}

La educación ambiental tiene un papel importante en los currículos de las instituciones de educación superior, esto atendiendo a la necesidad de que los futuros tecnólogos adquieran competencias básicas para tomar decisiones y realizar sus acciones profesionales desde la perspectiva de la sostenibilidad, teniendo en cuenta los ejes cognitivos (conocer y comprender) y actitudinal (saber actuar). De acuerdo a Aznar Minguet (2006) la racionalidad teórica (cognitivo) permite aplicar conocimiento científico para mejorar la práctica de la educación para la sostenibilidad; y la racionalidad ética (actitudes) que involucra en la propia definición de desarrollo humano ambientalmente sostenible la concepción moral y la actitud ética desde la que fomentar los valores de la sostenibilidad. 
Número Extraordinario. p.p. $135-144$

Memorias del Primer encuentro ambiental Universidad, ambiente y sustentabilidad: experiencias y prácticas.

De la misma manera es necesario tener en cuenta la visión holística, integral e interdisciplinaria como medio para hacer un cambio en la educación superior y para la inclusión de las competencias para el desarrollo sostenible en el currículo. Esta investigación espera hacer una propuesta curricular para un espacio académico pero es necesario que se trascienda a los límites de lo interdisciplinario para superar la sectorización del conocimiento, para que el desarrollo humano sostenible pueda abordarse desde diferentes disciplinas, pero no por sí solas, ya que es muy poco lo que aportan los equipos multidisciplinarios si los expertos de cada disciplina ofrecen sólo una visión técnica de su especialidad, sin articular el conocimiento desde múltiples perspectivas.

Trabajar la educación ambiental en función del desarrollo sostenible (DS) es asumir una perspectiva más crítica, analítica y participativa de los problemas socio-ambientales, más allá de lo que puede lograrse con una visión naturalista adoptada comúnmente en los espacios universitarios en típicos modelos clásicos; es decir no se trata únicamente de reconocer la sostenibilidad desde lo ambiental sino desde donde el estudiante tenga una posición activa frente al conocimiento, las habilidades y valores y sea capaz de generar cambios en la vida natural y social actual a favor del ambiente sin comprometer las condiciones futuras.

De la misma manera esta propuesta curricular será un medio necesario para la introducción a las realidades globales el desarrollo sostenible así como los temas educativos que faciliten el contacto con los problemas socioambientales en el propio escenario en el que ocurren; estas vivencias habrían de contemplar el análisis y la reflexión crítica sobre las alternativas de intervención en las relaciones entre la sociedad y el medio, orientando, propiciando y defendiendo un desarrollo humano ambiental y socialmente sostenible en el plan de estudios.

Se espera entonces que el desarrollo de las acciones conjuntas e integradas en la propuesta curricular se fomente por medio de grupos de trabajo universitarios que coordinen y potencien dicho proceso no sólo a un programa si no a nivel de toda la institución. 
Bio-grafía. Escritos sobre la Biología y su Enseñanza. ISSN 2027-1034

Número Extraordinario. p.p. $135-144$

Memorias del Primer encuentro ambiental Universidad, ambiente y sustentabilidad: experiencias y prácticas.

\section{REFERENCIAS BIBLIOGRÁFICAS}

Aznar Minguet, P. (2006). El reto educativo de la sostenibilidad en el marco del espacio europeo de la educación superior. En A. Escolano Benito, Educación superior y desarrollo sostenible. Madrid: Biblioteca Nueva.

Murga-Menoyo, M. A. (2015). Competencias para el desarrollo sostenible: las capacidades, actitudes y valores meta de la educación en el marco de la Agenda global post-2015. Foro de Educación, 13(19), 5583. doi: http://dx.doi.org/10.14516/fde.2015.013.019.004

Steanhouse, L. (1985). Research as a basis for teaching Heinemann educational. Londres: Books. Ltd.

Sauvé, L. (2010). Educación científica y educación ambiental: un cruce fecundo. Enseñanza de las ciencias, 28(1), 5-18.

UNESCO (2014a). Documento de posición sobre la educación después de 2015. ED-14/EFA/ POST-2015/1. Recuperado de http://unesdoc.unesco.org/ images/0022/002273/227336s.pdf

UNESCO (2014b). Roadmap for Implementing the Global Action Programme on Education for Sustainable Development. Recuperado de http://unesdoc. unesco.org/images/0023/002305/230514e.pdf 